Estudios Geológicos, 65(1)

enero-junio 2009, 67-77

ISSN: 0367-0449

doi:10.3989/egeol.39591.047

\title{
Inversión de efectos de sitio y factor $Q$ utilizando cocientes espectrales
}

\author{
Inversion of site effects and $\mathrm{Q}$ factor using spectral ratios
}

\author{
A. Moya Fernández 1
}

RESUMEN

En este estudio se llevó a cabo la inversión de los efectos de sitio y del valor de la atenuación $Q$, utilizando la técnica de cocientes espectrales. La zona de estudio corresponde a la parte central de Costa Rica y los datos utilizados provienen de la red de movimiento fuerte que el Laboratorio de Ingeniería Sísmica (INII-UCR) tiene instalada en el país. La técnica empleada utiliza un evento como referencia en lugar de una estación sísmica, por esa razón, los efectos de sitio que se obtienen son los valores absolutos de amplificación. Los resultados muestran que varias de las estaciones ubicadas en sitios sobre suelo blando tienen mayores factores de amplificación que las que se encuentran sobre terrenos relativamente mejor consolidados. También, el valor de la atenuación en la forma de factor $Q$ se encontró que era dependiente de la frecuencia con un valor aproximado de $Q(f)=(131,6 \pm 0,1) f^{(1,1 \pm 0,2)}$.

Palabras clave: Terremotos, Efectos de sitio, Atenuación, Amplificación, Factor Q, Costa Rica.

\begin{abstract}
Site effects and $Q$ factor were inverted using the spectral ratio technique. The study area corresponds to central Costa Rica where the Earthquake Engineering Laboratory (INII-UCR) runs a strong motion network. The technique differs from the usual approach in that it makes use of a reference earthquake instead of a reference site. For that reason, the amplitude of the site effects that are obtained corresponds to the absolute amplification. The results indicate that stations located on soft sediment sites tend to have larger amplification values compared to other stations located on more compacted soils. The $Q$ factor we obtained was frequency dependent with an approximate value of $Q(f)=(131,6 \pm 0,1) f^{(1,1 \pm 0,2)}$.
\end{abstract}

Key words: Earthquakes, Site effects, Attenuation, Amplification, Q factor, Costa Rica.

\section{Introducción}

Cuando ocurre un terremoto, las ondas sísmicas se propagan desde el foco o hipocentro en todas las direcciones a través de lo que se denomina el medio de propagación. Este medio está formado por todos los estratos e irregularidades que existen bajo la superficie de la tierra y cuyo efecto sobre la señal se manifiesta en una modificación de su contenido frecuencial y de su amplitud. La señal va perdiendo energía conforme avanza hacia la superficie. Sin embargo, es precisamente cerca de la superficie cuando esta situación se invierte. Cerca de la superficie la amplitud tiende a aumentar producto de la baja impedancia característica de cuencas sedimentarias, zonas de relleno y valles aluviales por ejemplo. Los sitios en roca, tales como afloramientos de intrusivos, calizas y lavas no producen una amplificación tan importante como es el caso de materiales menos consolidados, pero cierto nivel de amplificación ha sido detectado en tales ambientes (Steidl et al., 1996). A este fenómeno se le denomina efecto de sitio y modifica el registro sísmico en su contenido frecuencial, amplitud y duración.

Debido a esa complejidad del registro sísmico, una de las técnicas utilizadas para separar la fuente, el medio y los efectos de sitio es el de los cocientes espectrales. En el caso de los efectos de sitio, se uti-

\footnotetext{
1 Laboratorio de Ingeniería Sísmica, Instituto de Investigaciones en Ingeniería, Universidad de Costa Rica.

Email: aaronmoya@gmail.com
} 
lizan señales de un mismo sismo registrado en dos o más estaciones. Al efectuarse el cociente espectral, el factor de la fuente sísmica se cancela automáticamente por ser común a ambas estaciones. De esta forma, se pueden escribir una serie de ecuaciones que luego se ordenan en forma matricial para invertir los datos y obtener un resultado. La ventaja de dicha inversión es que es lineal, lo que significa que el resultado es único aunque éste dependerá de la cantidad de información con la que se cuente.

Es importante poder determinar con exactitud los valores de la amplificación del suelo y de la atenuación del medio tanto si se quiere estudiar la fuente sísmica como evaluar el potencial amplificador de un sitio sobre el cual se encuentra algún tipo de asentamiento humano. En Costa Rica se han realizado diversos estudios tendentes a cuantificar el nivel de amplificación del suelo principalmente en la capital y ciudades vecinas (lo que se conoce como el Gran Area Metropolitana, GAM) (fig. 1).

Ramírez et al. (1996) utilizaron un modelo de descripción de la geología superficial para analizar la amplificación en la parte central del país donde se encuentran cuatro de las ciudades más pobladas: San José, Alajuela, Cartago y Heredia (fig. 1). Las simulaciones que realizaron dieron como resultado que las ciudades de Alajuela y Cartago eran las que presentaban mayor amenaza de amplificación. Laporte (1994) utilizó un perfil típico de suelos obtenidos de la descripción de pozos de la ciudad de Cartago para evaluar el nivel de amplificación. Sus resultados revelaron que en Cartago la amplificación dinámica llegó a ser de hasta tres veces superior cerca de los picos fundamentales de los estratos $(2,5$ y $5,0 \mathrm{~Hz})$ cuando se simularon los sismos de Piedras Negras del 22 de diciembre de 1990 (6,0 Mw a 48,5 km de distancia la ciudad de Cartago) y el del Valle de la Estrella (Telire, también conocido como el Terremoto de Limón) el 22 de abril de $1991(7,7 \mathrm{Mw}$ a 92,0 km de distancia de la ciudad de Cartago) (fig. 2). Un estudio de microzonificación sísmica de Schmidt et al. (2005) también abordó nuevamente la determinación de los efectos de amplificación del suelo en el GAM mediante la realización de pruebas geofísicas y mapas de isoperiodos entre otros. En ese trabajo se determinaron los efectos de sitio usando ruido ambiente y tomando el cociente de la componente horizontal sobre la vertical usando sismos. También se estimó el cociente espectral de señales obtenidas en sedimentos con respecto a sitios en roca, pero usando sismos lejanos, de tal forma que el efecto de la atenua- ción se cancelara junto con el de la fuente sísmica. De acuerdo a los resultados obtenidos, la amplificación de la señal es mayor en la parte noreste y oeste de la capital en donde el espesor del material no consolidado (material volcánico como flujos piroclásticos y ceniza) alcanza profundidades de hasta $40 \mathrm{~m}$ hasta llegar a un lahar donde la velocidad de la onda $S$ es superior a los $500 \mathrm{~m} / \mathrm{s}$.

Todos estos estudios han tratado de caracterizar el fenómeno de la amplificación en forma independiente al de la atenuación. En esta investigación, por el contrario, el objetivo es determinar tanto los efectos de sitio como la atenuación usando la técnica de los cocientes espectrales utilizada por Iwata \& Irikura (1986) con las modificaciones propuestas por Moya \& Irikura (2003). También se identificarán los periodos fundamentales de cada sitio involucrado en la inversión así como el factor de amplificación del mismo.

\section{Red de movimiento fuerte}

Este trabajo se realizó con los datos de la red de movimiento fuerte (fig. 1) del Laboratorio de Ingeniería Sísmica (LIS) adscrito al Instituto de Investigaciones en Ingeniería (INII) de la Universidad de Costa Rica. La mayoría de las estaciones acelerográficas se ubican en el centro del país y a lo largo de la costa pacífica ya que se trata de zonas densamente pobladas y de importante actividad sísmica. Las estaciones RMOI, RTUR, RALT, RGAR y RBAR también forman parte de la red del LIS aunque son propiedad de la Refinadora Costarricense de Petróleo (RECOPE) y se encuentran a lo largo del poliducto que va desde la costa del Caribe hasta la costa del Pacífico.

La red ha estado en funcionamiento desde principios de la década de 1990. En sus inicios los aparatos eran todos del tipo analógico SMA1 de Kinemetrics. Con el paso del tiempo y la llegada de los aparatos digitales, se empezó a hacer una sustitución gradual del equipo analógico que para el año 2004 se vio finalizada. El 20 de noviembre de ese mismo año ocurrió un sismo de $6,4 \mathrm{Mw}$ cuyo epicentro estuvo cerca de un pueblo llamado Damas y la red, para entonces formada por aparatos tipo ETNA, K2, SSA2 y QDR (todos ellos de Kinemetrics), registró varias de sus réplicas (tablas 1 y 2).

La forma en que operan los instrumentos es mediante la activación de un nivel de disparo predefinido. Si la aceleración excede cierto umbral, el 


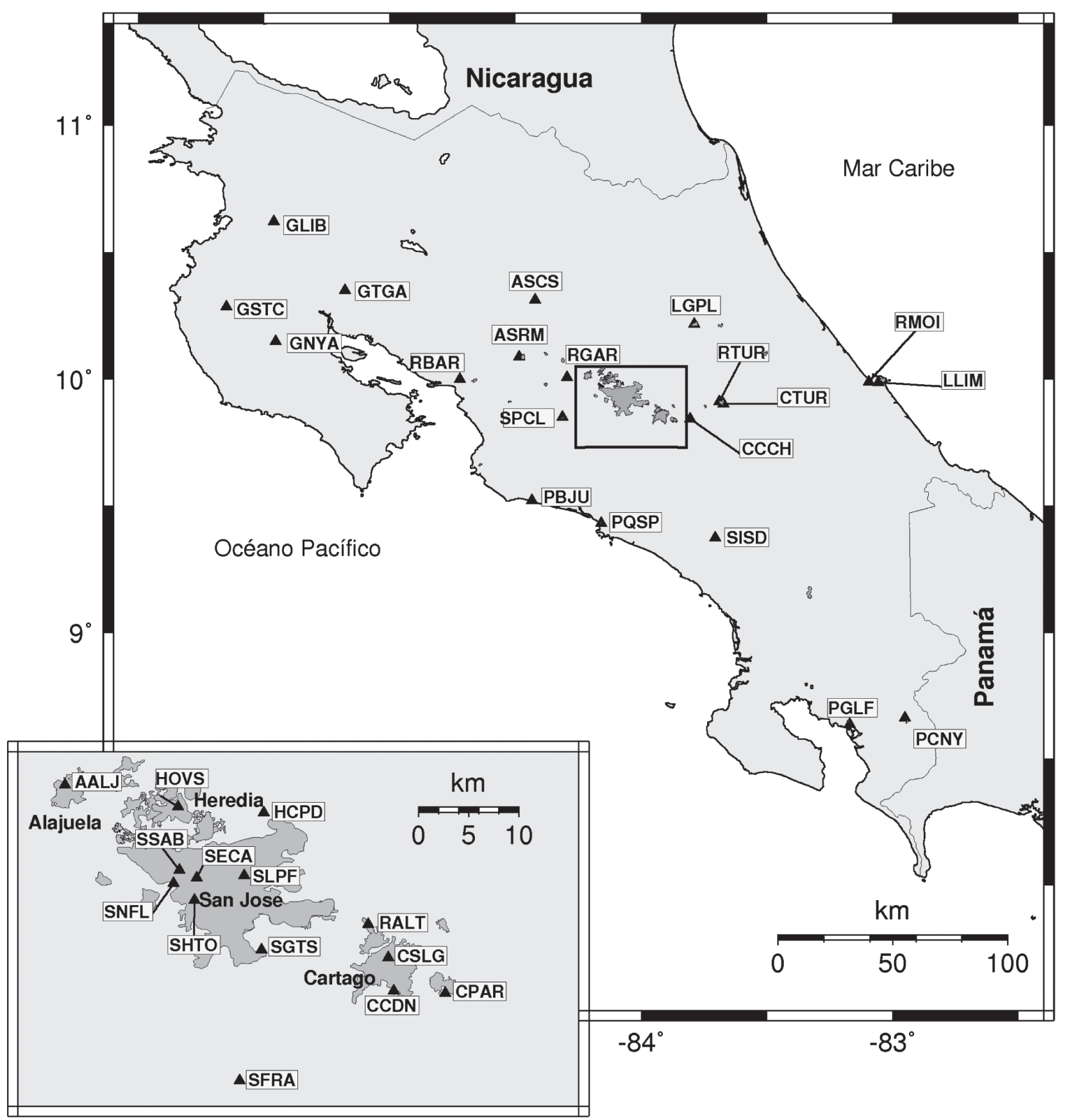

Fig. 1.-Ubicación de las estaciones (triángulos) acelerográficas del Laboratorio de Ingeniería Sísmica (INII-UCR). Las estaciones cuyo nombre inicia con «R» son propiedad de la Refinería Costarricense de Petróleo (RECOPE) pero operadas por el LIS. En el recuadro aparecen los principales centros poblacionales de la zona central: San José, Alajuela, Cartago y Heredia.

aparato guarda la información, si ese nivel no es excedido, el registro no se guarda. Por esa razón, no todos los aparatos poseen el mismo número de eventos y muchas de las estaciones ubicadas lejos de la capital poseen poca información que pudiera ser contemplada en este análisis (tabla 2). Por ejemplo, no se pudo utilizar ningún sismo de la zona norte del país (estaciones GTGA, GNYA, GSTC y GLIB) ya pocos de ellos fueron registrados en el resto de las estaciones en la zona central y sur de Costa Rica.

La figura 2 muestra la distribución de las estaciones utilizadas en la inversión y la ubicación de los 


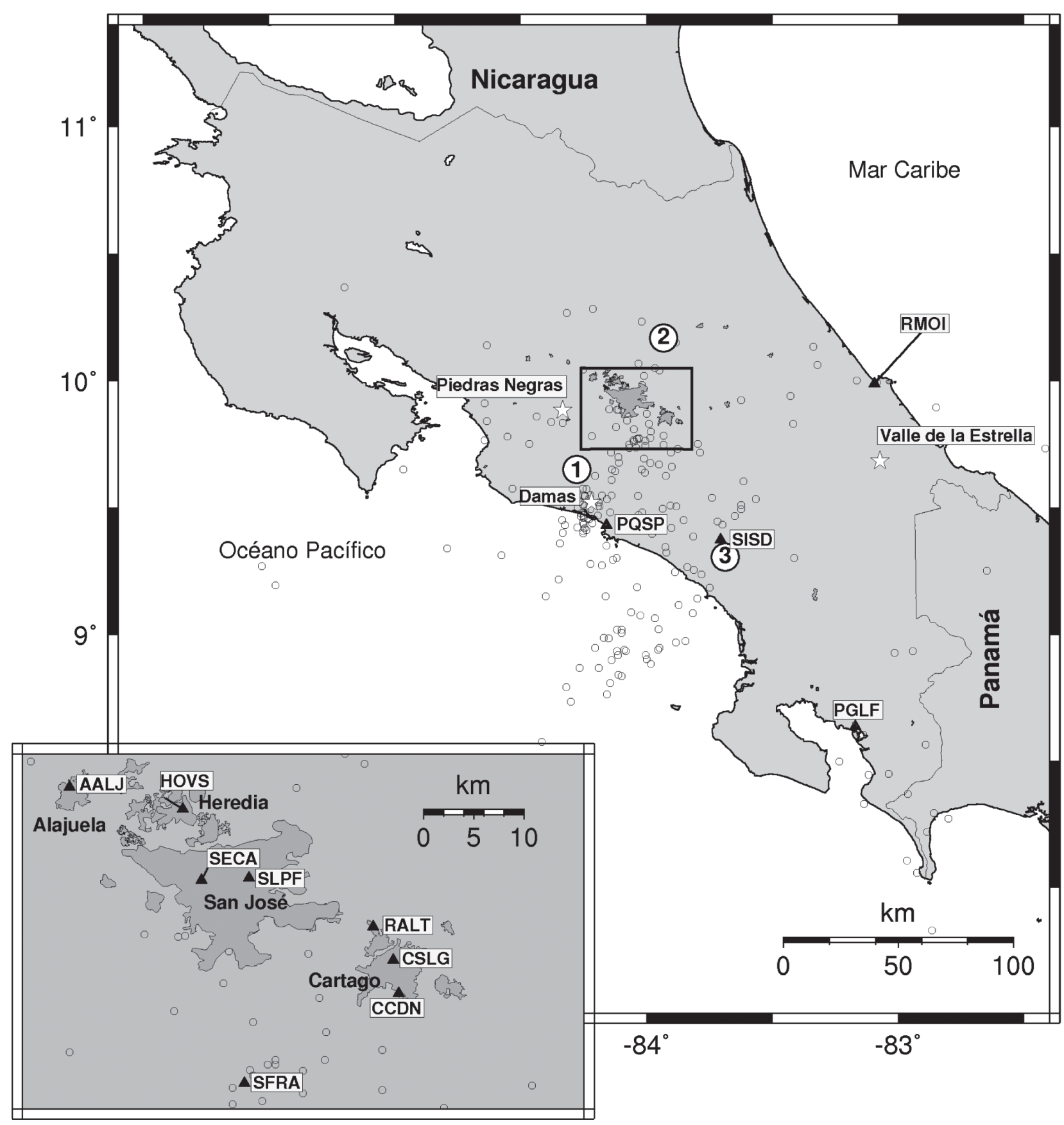

Fig. 2.-Distribución espacial de los eventos (círculos) y estaciones (triángulos) acelerográficas utilizadas en este estudio. Los círculos denotados con números corresponden a los eventos usados como referencia cuyos parámetros se muestran en la tabla 1 . Los eventos mostrados con estrellas son los del 22 de diciembre de 1990 en Piedras Negras (6,0 Mw), el 22 de abril de 1991 en el Valle de la Estrella (7,7 Mw) y el del 20 de noviembre de 2004 en Damas (6,2 Mw).

epicentros de los eventos con Mw superior a 3,0 (tabla 2). Los parámetros de ubicación de los eventos fueron obtenidos de la Red Sismológica Nacional (RSN-ICE) y del Observatorio Vulcanológico y Sismológico Nacional (OVSICORI-UNA).
Muchos de los eventos proceden de la estación CCDN, SISD y SECA que han contado con aparatos digitales del tipo SSA2 desde que empezaron a funcionar. El instrumento en la estación SLPF es el único del tipo K2 que formaba parte de una red local de 
Tabla 1.-Número de registros, geología superficial y tipo de instrumento acelerográfico en las estaciones seleccionadas para este estudio. Registros totales son los registrados por la estación y registros usados son los utilizados en este estudio

\begin{tabular}{|c|c|c|c|c|}
\hline \multirow[b]{2}{*}{ Estación } & \multicolumn{2}{|c|}{ Número de registros } & \multirow[b]{2}{*}{ Geología superficial } & \multirow{2}{*}{$\begin{array}{c}\text { Tipo de } \\
\text { instrumento }\end{array}$} \\
\hline & Totales & Usados & & \\
\hline AALJ & 7 & 7 & Lahares (brechas, arenas y arcillas) & ETNA \\
\hline SISD & 15 & 10 & Lutitas, areniscas y conglomerados & SSA2 \\
\hline SLPF & 52 & 22 & Lahares (brechas, arenas y arcillas) & K2 \\
\hline CCDN & 35 & 29 & Rocas calizas, areniscas y lutitas de origen aluvial y coluvial & SSA2 \\
\hline CSLG & 18 & 15 & Lahares (brechas, arenas y arcillas) & ETNA \\
\hline HOVS & 12 & 12 & No disponible & SSA2 \\
\hline SECA & 20 & 20 & Lahares (brechas, arenas y arcillas) & SSA2 \\
\hline RMOI & 14 & 7 & Areniscas, conglomerados y arrecifes coralinos & ETNA \\
\hline PQSP & 34 & 18 & Basaltos y aglomerados de basaltos & ETNA \\
\hline SFRA & 91 & 36 & Andesitas y flujos piroclásticos & ETNA, QDR \\
\hline RALT & 8 & 8 & Andesitas, dacitas y tobas & ETNA \\
\hline PGLF & 12 & 4 & Brechas basálticas con sedimentos pelágicos incluidos & ETNA \\
\hline
\end{tabular}

movimiento fuerte llamada Red SAN (Moya et al., 1999). El resto de las estaciones de la tabla 1 poseen un aparato tipo ETNA y en algunas ocasiones tuvieron un aparato tipo QDR en forma temporal. Los aparatos QDR son convertidores analógico-digitales que se empezaron a usar una vez que los instrumentos SMA1 fueron descontinuados. La velocidad de muestreo de los QDR es de 100 muestras/s mientras que la de los K2, ETNA y SSA2 es de 200 muestras.

\section{Metodología}

Para el análisis, cada registro fue graficado y se leyeron los tiempos de arribo de las ondas $S$ en las componentes horizontales. A partir de ese punto, se tomó una ventana de tiempo de 3,0 s. La razón para utilizar solamente 3,0 s fue para evitar la contaminación del registro por la presencia de ondas superficiales.

Se aplicó un taper de tipo coseno con un ancho de ventana equivalente a $0,1 \mathrm{~s}$ antes y después de la ventana de 3,0 s y se calculó la transformada de Fourier de los espectros de aceleración. Cada espectro fue luego dividido por el factor $(2 \pi f)^{2}$ para obtener el espectro de desplazamiento. Luego se calculó la resultante de la suma vectorial de los espectros de la componente horizontal, $O(f)$, es decir

$$
O(f)=\sqrt{\left[N S(f)^{2}+E W(f)^{2}\right]}
$$

donde $N S(f)$ y $E W(f)$ y son los espectros de amplitud de las componentes norte-sur y este-oeste respectivamente.
Los detalles del procedimiento de la inversión se explican posteriormente en Moya \& Irikura (2003), por lo que aquí se mostrará solamente la parte más importante de la metodología utilizada. Un espectro de amplitud de un sismo $i$ observado en un sitio $j$ en el dominio de la frecuencia, adquiere la forma

$$
O_{i j}(f)=S_{i}(f) G_{j}(f) R_{i j}^{-1} e^{\frac{-\pi f R_{i j}}{Q(f) \beta}}
$$

donde $S_{i}(f)$ es el espectro de la fuente del sismo $i$, $G_{j}(f)$ es el espectro del efecto de sitio de la estación $j, R_{i j}^{-1}$ es el factor de corrección por distancia hipocentral del evento $i$ en la estación $j, Q(f)$ es el factor de calidad y $\beta$ es la velocidad de las ondas $\mathrm{S}$. Si ese espectro de la estación $j$ se divide entre otro del mismo evento pero en diferente estación, digamos $r$, se obtiene un cociente espectral de la forma,

$$
\frac{O_{i j}(f)}{O_{i r}(f)}=G_{r}^{j}(f) R_{i j}^{i r} e^{\frac{-\pi f\left(R_{i j}-R_{i r}\right)}{Q(f) \beta}}
$$

Que puede ser luego linearizada tomando logaritmos a ambos lados

$$
\ln \left[G_{j}(f)\right]-\ln \left[G_{r}(f)\right]-\frac{\pi f\left(R_{i j}-R_{i r}\right)}{Q(f) \beta}=\ln \left[O_{i r}^{i j}(f) R_{i r}^{i j}\right]
$$

La ecuación como tal no se puede utilizar, ya que tanto $G_{j}$ como $G_{r}$ son parámetros que deben ser determinados, lo mismo que el factor $Q$ y no existe ningún sitio de referencia. Es decir, la solución no es única ya que para solucionar la ecuación 4 es necesario hacer alguna de las siguientes suposiciones:

1. Asumir un valor para el sitio $G_{j}$ y resolver para $G_{r}$ y $Q$. 
Tabla 2.-Parámetros de ubicación de los sismos usados en este estudio

\begin{tabular}{|c|c|c|c|c|}
\hline Fecha:hora(UTC) & Long. & Lat. & $\mathrm{Mw}$ & Prof. (km) \\
\hline 19910803:1322 & -84.000 & 9.870 & 3,8 & 13,8 \\
\hline 19910806:1055 & -84.037 & 9.770 & 4,7 & 5,2 \\
\hline 19910809:0940 & -84.005 & 9.764 & 4,1 & 7,0 \\
\hline 19980930:1859 (1) & -83.933 & 10.167 & 4,3 & 20,0 \\
\hline 19990416:2350 & -84.250 & 9.433 & 4,7 & 37,0 \\
\hline 19990820:1002 & -84.150 & 8.983 & 6,5 & 22,0 \\
\hline 20000120:1220 & -83.883 & 10.150 & 4,5 & 17,0 \\
\hline $20000721 ; 0153$ & -85.475 & 9.193 & 6,2 & 25,6 \\
\hline 20000902:0143 & -83.782 & 9.235 & 5,2 & 51,5 \\
\hline 20001109:0814 & -83.698 & 9.432 & 4,3 & 7,9 \\
\hline 20001117:1952 & -83.625 & 9.509 & 4,3 & 16,9 \\
\hline 20001209:2032 & -84.080 & 9.957 & 2,9 & 14,0 \\
\hline 20001229:0845 & -84.112 & 9.698 & 4,5 & 60,0 \\
\hline 20010130:0431 & -84.060 & 9.938 & 4,2 & 5,2 \\
\hline 20010130:0431 & -84.060 & 9.938 & 4,2 & 5,2 \\
\hline 20010130:2304 & -84.076 & 9.945 & 3,8 & 5,4 \\
\hline 20010130:2304 & -84.076 & 9.945 & 3,8 & 5,4 \\
\hline $20010224: 2340$ & -83.625 & 9.923 & 4,8 & 12,7 \\
\hline 20010402:0249 & -83.812 & 9.253 & 4,0 & 41,1 \\
\hline 20010405:0409 & -83.877 & 9.731 & 3,9 & 15,9 \\
\hline 20010412:1233 & -83.872 & 9.115 & 5,0 & 39,0 \\
\hline 20010715:1934 & -84.139 & 9.609 & 4,7 & 26,5 \\
\hline 20020122:0435 & -84.110 & 9.677 & 3,8 & 16,7 \\
\hline 20020602:1252 (2) & -83.688 & 9.904 & 4,9 & 26,7 \\
\hline 20020731:0017 & -82.867 & 7.828 & 6,2 & 20,0 \\
\hline 20030916:2003 & -84.378 & 9.837 & 5,4 & 54,9 \\
\hline 20030916:2003 & -84.378 & 9.837 & 5,4 & 54,9 \\
\hline 20031017:0007 & -83.797 & 9.751 & 4,2 & 10,0 \\
\hline 20031225:0711 & -82.800 & 8.271 & 6,6 & 26,0 \\
\hline 20040102:2202 & -84.007 & 9.638 & 4,8 & 74,1 \\
\hline 20040107:1042 & -82.886 & 8.218 & 5,5 & 15,0 \\
\hline 20040204:1159 & -83.136 & 8.328 & 5,8 & 14,0 \\
\hline 20040407:2023 & -84.089 & 8.938 & 5,5 & 6,6 \\
\hline 20040726:1958 & -83.922 & 9.323 & 4,3 & 49,0 \\
\hline 20040726:1958 & -83.922 & 9.323 & 4,3 & 49,0 \\
\hline 20040817:1346 & -84.215 & 9.437 & 4,1 & 40,0 \\
\hline 20041120:0807 & -84.220 & 9.520 & 6,2 & 25,0 \\
\hline 20041120:0807 & -84.220 & 9.520 & 6,2 & 25,0 \\
\hline 20041120:0814 & -84.160 & 9.350 & 4,3 & 4,3 \\
\hline 20041120:0816 & -84.120 & 9.300 & 3,7 & 12,0 \\
\hline 20041120:0816 & -84.120 & 9.300 & 3,7 & 12,0 \\
\hline 20041120:0904 & -84.266 & 9.522 & 4,0 & 12,1 \\
\hline 20041120:1215 & -84.193 & 9.468 & 4,6 & 3,9 \\
\hline 20041120:1408 & -84.275 & 9.422 & 4,4 & 2,7 \\
\hline 20051228:1527 (3) & -84.278 & 9.650 & 5,5 & 43,0 \\
\hline 20061118:1913 & -84.138 & 9.650 & 3,4 & 33,4 \\
\hline
\end{tabular}

Los datos fueron obtenidos de la Red Sismológica Nacional (RSN-ICE) y del Observatorio Vulcanológico y Sismológico de Costa Rica (OVSICORI-UNA).

2. Asumir un valor para el sitio $G_{r}$ y resolver para $G_{j}$ y $Q$.

3. Resolver la ecuación de la forma convencional en la que la solución da un valor de amplitud relativo y no absoluto de la forma $G_{j}(f) / G_{r}(f)$.

En la metodología de este estudio se optó por utilizar un evento sísmico para el cual se asume que se conocen los valores del momento sísmico y la frecuencia de esquina. A este evento se le denomina evento de referencia y se caracteriza porque su espectro es un espectro de Brune (1970) y sigue un modelo omega-cuadrado (Aki, 1967) tal y como lo muestra la ecuación 5.

$$
S_{i}(f)=\frac{\Omega_{0_{i}}}{1+\left(\frac{f}{f_{0_{i}}}\right)^{2}}
$$

donde $\Omega_{0_{i}}$ es el nivel plano de la baja frecuencia y $f_{0_{i}}$ es la frecuencia de esquina del evento $i$. En la ecuación 6 , el evento de referencia es un término conocido, pero no lo son el efecto de sitio ni el valor $Q$. Esta ecuación se puede entonces añadir al final de la ecuación 4 en una forma matricial y realizar una sola inversión del conjunto para obtener los efectos de sitio absolutos.

$$
\ln \left[G_{j}(f)\right]-\frac{\pi f\left(R_{i j}\right)}{Q(f) \beta}=\ln \left[O_{i j}(f) R_{i j}\right]-\ln \left[S_{i}(f)\right]
$$

La suposición de que el momento sísmico y la frecuencia de esquina son conocidos es importante para obtener el valor de amplificación total de cada sitio. Una sobreestimación del nivel plano resultaría en una subestimación de la amplitud de los efectos de sitio y una subestimación de ese nivel en una sobreestimación de dichos efectos. En este estudio, se seleccionaron tres eventos que fueron registrados por la mayoría de las estaciones para ser utilizados como eventos de referencia. La ubicación de esos eventos se muestra en la figura 2 con los números (1), (2) y (3) que corresponden a los eventos señalados en la tabla 1: 19980930:1859, 20020602:1252 y 20051228:1527 respectivamente.

Para determinar el valor del nivel plano de la baja frecuencia del espectro se procedió de la siguiente manera. Los espectros de desplazamiento de cada evento se graficaron para todas las estaciones que los habían registrado después de haberlos corregido por el factor de distancia hipocentral (fig. 3). Bajo la suposición de que todos los sitios deben amplificar la señal original, se tomó como el nivel plano del espectro de la fuente el valor de la asíntota inferior. La asíntota inferior representa entonces una aproximación a un valor de momento sísmico que debería corresponder a aquel que aún no se ha visto influenciado por el efecto amplificador de cada sitio, una vez removido el efecto de superficie libre. Los valores de nivel plano leídos de esta manera y el efecto de superficie libre se resumen en la tabla 3. 
1998/09/30-1859

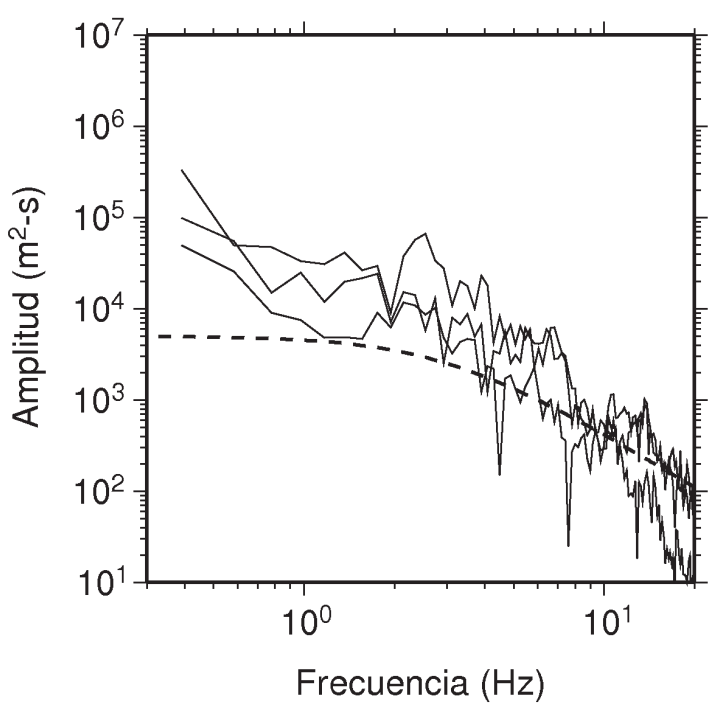

2005/12/28-1527

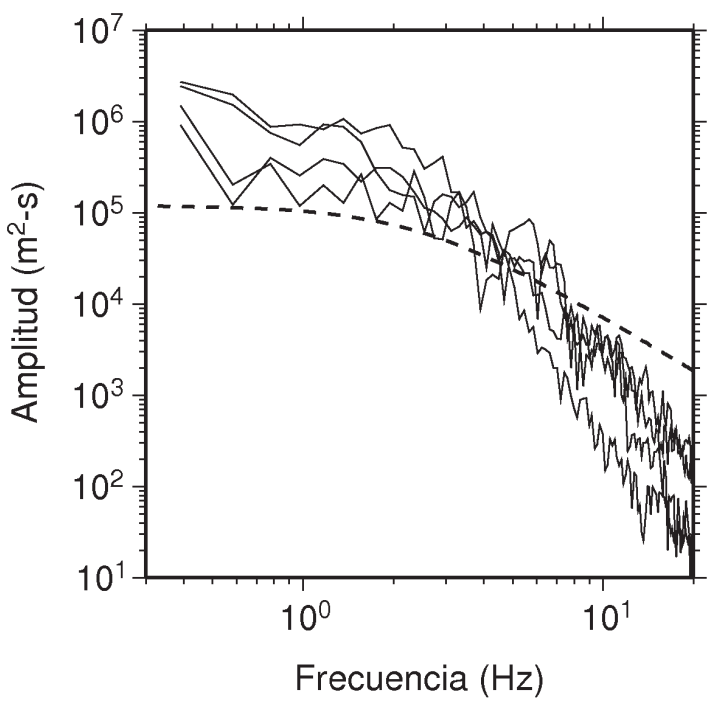

2002/06/02-1252

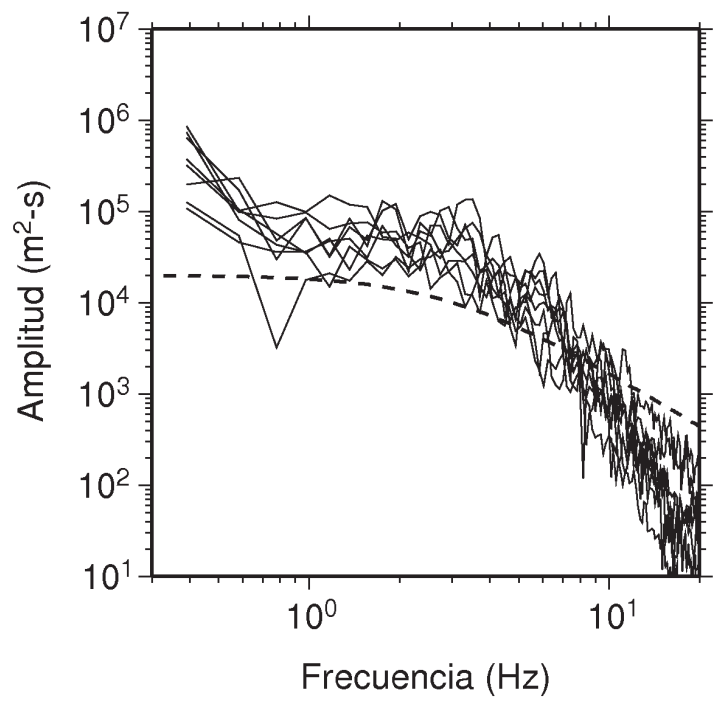

Fig. 3.-Determinación del nivel plano utilizando el valor de la asíntota inferior del conjunto de espectros. La línea punteada corresponde al modelo omega-cuadrado que se ajustó a los datos.

La frecuencia de esquina se determinó leyendo el valor del espectro de desplazamiento de tal forma que el modelo omega cuadrado se ajustara lo mejor posible a los espectros observados tal y como se muestra en la figura 3. En el caso del evento (1) fue de 3,0, en el evento (2) fue de 3,0 y el del evento (3) $2,5 \mathrm{~Hz}$ (tabla 3). Debido a que se están utilizando tres eventos como referencia se esperaría que el valor de la incertidumbre no dependiera de un solo evento sino que se distribuyera entre los tres eventos usados como referencia (Moya \& Irikura, 2003).

Habiéndose determinado el valor del nivel plano y la frecuencia de esquina, se procedió a escribir los cocientes espectrales en forma matricial. La matriz 
Tabla 3.-Valores del nivel plano estimado usando la asíntota inferior para los eventos usados como referencia. Se dan también los valores de superficie libre y frecuencia de esquina $\left(f_{e}\right)$ así como el número de registros usados en la inversión

\begin{tabular}{cccccc}
\hline & & & & & $\begin{array}{c}\text { Núm. de } \\
\text { registros } \\
\text { Núm. }\end{array}$ \\
\hline 1 & Fecha & Asíntota & Sup. libre & $\mathrm{f}_{\mathrm{e}}$. & usados \\
\hline 2 & $20020602: 1252$ & $2 \times 10 * * 4$ & $1 \times 10 * * 4$ & 3,0 & 7 \\
3 & $20051228: 1527$ & $1 \times 10 * * 5$ & $5 \times 10 * * 4$ & 2,5 & 4 \\
\hline
\end{tabular}

para la inversión consta de dos partes. La primera es la que contiene los cocientes espectrales de los efectos de sitio y la segunda los modelos de fuente sísmica

$$
\left[\begin{array}{l}
A \\
c
\end{array}\right] \cdot[x]=\left[\begin{array}{l}
b \\
d
\end{array}\right]
$$

En donde $A$ es el conjunto de todos los cocientes dados por la ecuación 4 y $c$ a los dados por la ecuación 6. $x$ contiene los valores de los efectos de sitio y el factor $Q$ que nos interesa encontrar. $b$ y $d$ son los términos de la parte de la derecha de las ecuaciones 4 y 6 respectivamente. Al tratarse de un caso lineal, podemos utilizar la técnica de la descomposición del valor singular (singular value decomposition) frecuencia a frecuencia.

\section{Discusión y resultados}

De acuerdo con el resultado de la inversión que se muestra en la figura 4, todos los sitios estudiados presentan niveles de amplificación importantes. Esto incluye sitios como PGLF, PQSP y CCDN que, en relación al resto, se encuentran ubicados sobre un tipo de suelo que podría caracterizarse como roca (tabla 1). El espectro de PGLF es mucho más irregular que el de CCDN y PQSP. El pico máximo de PGLF se da a los 3,0 Hz.

AALJ junto a CSLG y SECA son las estaciones que mayor amplifican la señal en el rango de 0,3 a 4,0 Hz. Existen dos picos importantes en AALJ a los 1,0 y 2,5 Hz. En el caso de CSLG se da un pico a los 2,5 Hz que coincide con uno de los picos reportado por Laporte (1994) en sus simulaciones. Lo mismo sucede en CCDN, aunque esta estación se encuentra mucho más al sur y sobre un tipo de geología diferente a la de CSLG (tabla 1). El segundo pico reportado por Laporte (1994) a los 5,0 Hz no se pudo identificar por medio de esta técnica. En el caso de la estación SECA, al oeste de San José, los valores máximos ocurren a los 1,0, 2,5 y 4,0 Hz. Esta estación es la que se encuentra más cerca de uno de los puntos en los que Schmidt et al. (2005) identificaron espesores de suelo blando de hasta $30 \mathrm{~m}$ al oeste de San José.

La estación AALJ está ubicada cerca del parque central de la ciudad de Alajuela. En esta zona ocurrieron importantes daños a estructuras de 1 y 2 pisos durante el sismo de Piedras Negras en 1990. En esa estación también se registró el mayor valor de aceleración pico hasta la fecha, producto del mismo evento, y fue de $415 \mathrm{~cm} / \mathrm{s}^{2}$.

Resulta interesante comparar ese valor con el obtenido en la estación PQSP con el sismo de Damas en 2004. El evento de Piedras Negras tuvo una magnitud de 6,0 Mw y ocurrió a una distancia hipocentral de escasos $25 \mathrm{~km}$ de AALJ. El sismo de Damas tuvo una magnitud de 6,2 Mw y se localizó a $28 \mathrm{~km}$ de la estación PQSP. Mientras que en AALJ el valor máximo fue de $415 \mathrm{~cm} / \mathrm{s}^{2}$ en PQSP fue de $231 \mathrm{~cm} / \mathrm{s}^{2}$. Probablemente otros factores también influyan en estas diferencias como lo son posibles efectos de directividad de la fuente sísmica en el caso del sismo de Piedras Negras y falta de estos en el sismo de Damas (Pacheco et al., 2006), pero los efectos de sitio son claramente diferentes entre ambas estaciones, siendo mayor en AALJ.

Señales de sismos lejanos registrados tanto en AALJ como en SECA se caracterizan por poseer amplitudes comparativamente mayores a las de otras estaciones ubicadas en ciudades vecinas como HOVS y SLPF, esta última al este de San José (Moya et al., 1999; Schmidt et al., 2005). El caso de HOVS contrasta con el de AALJ tal y como se aprecia en la figura 4. Mientras que HOVS no posee valores pico de amplificación claramente definidos, en el caso de SLPF se observa uno a los 3,5 Hz.

Dos de las estaciones de RECOPE son RALT y RMOI. Los acelerógrafos se encuentran dentro de esos planteles cuyas estructuras son variables: tanques, tuberías, edificios. RALT se ubica en la zona central y RMOI en la costa Caribe. Las diferencias entre los efectos de sitio son claras. RALT posee menor amplificación que RMOI, pero mientras que RALT no posee picos claramente identificables, RMOI posee un valor de amplificación bastante notorio cercano a los 1,2 Hz. Este valor pico sería importante para eventos fuertes y lejanos, que son frecuentes en la costa pacífica de Costa Rica (DeShon et al., 2003; Husen et al., 2003; 


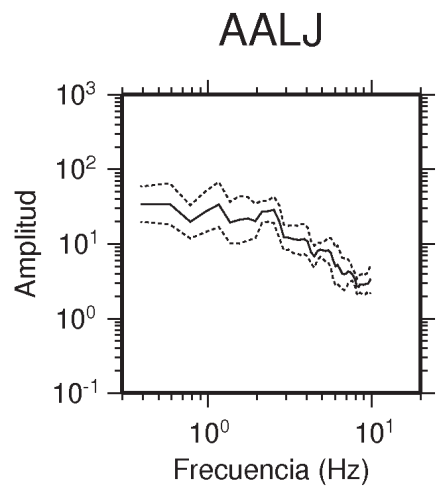

CSLG

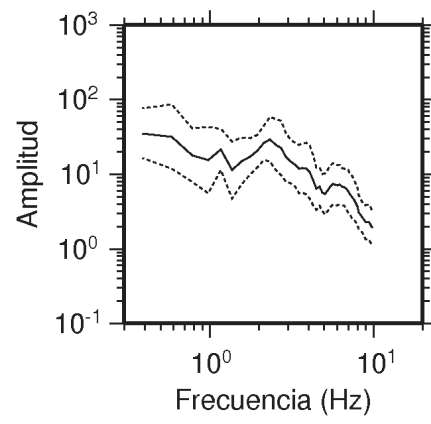

PQSP

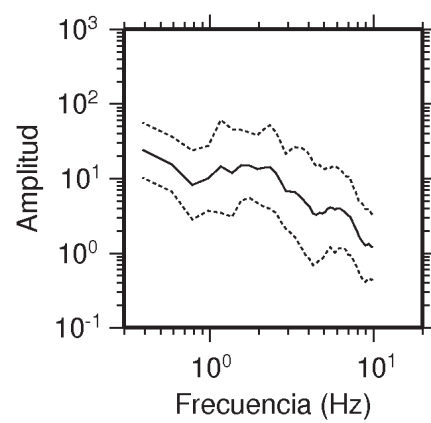

Frecuencia $(\mathrm{Hz})$
SISD

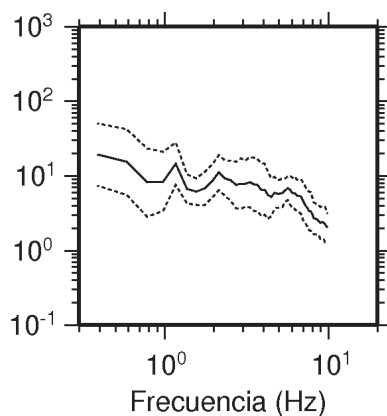

HOVS

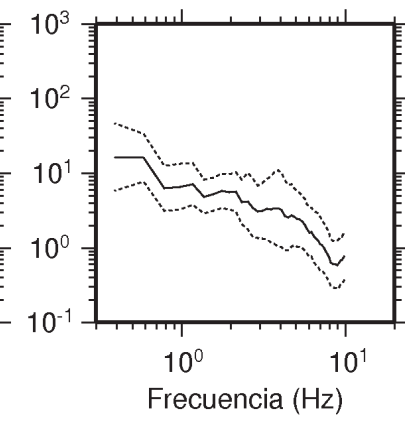

SFRA

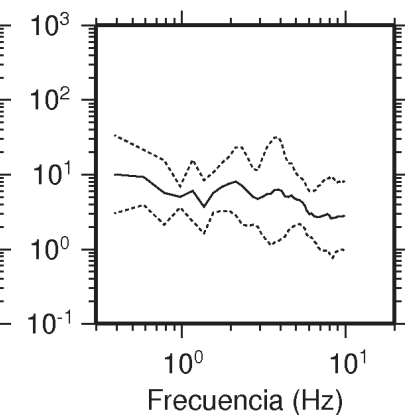

SLPF

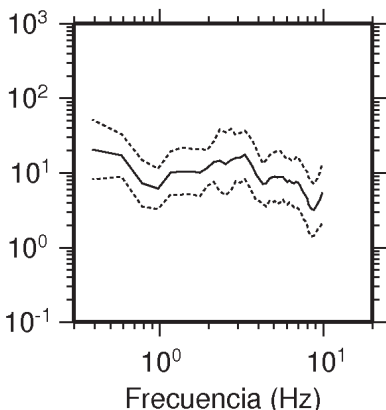

SECA

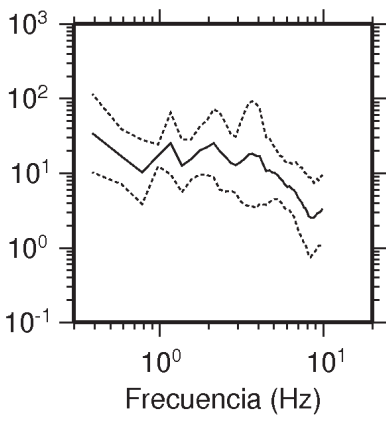

RALT

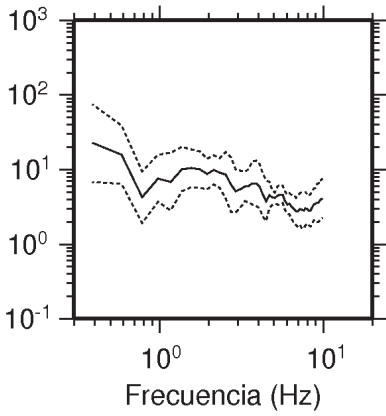

CCDN

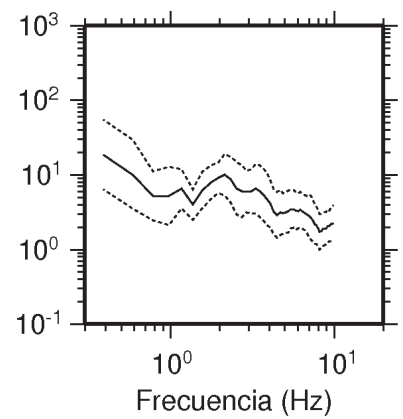

$\mathrm{RMOI}$

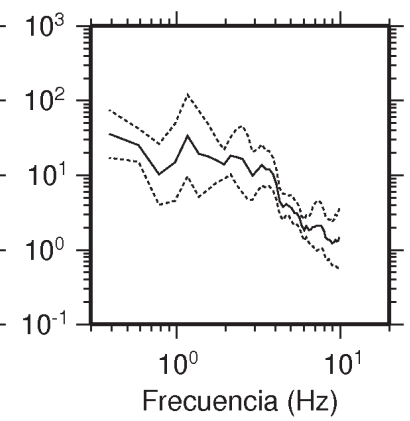

PGLF

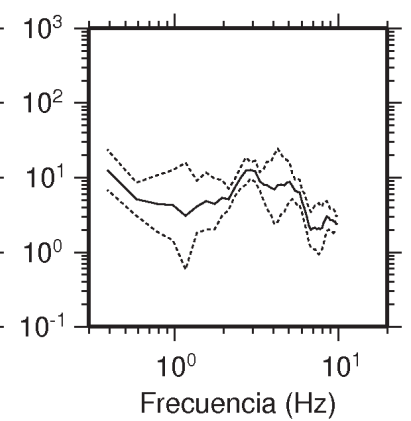

\section{$Q$ factor}

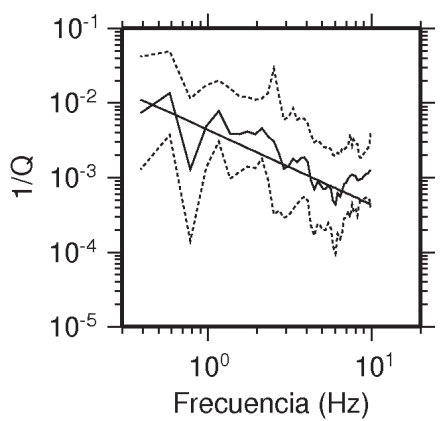

Fig. 4.- Resultado de la inversión de los cocientes espectrales y el factor $Q$. El valor promedio se da en la línea continua, mientras que se grafica la desviación estándar en líneas semicontinuas. Se muestra la recta de mejor ajuste del valor $Q$ obtenido. 
Protti et al., 1995), y que pueden excitar ese punto de vibración del suelo y tener repercusiones en estructuras elevadas o que contienen líquidos como lo son los tanques de almacenamiento de combustible.

SISD está ubicada al pie del valle de San Isidro de El General. Resulta interesante observar que la amplificación en SISD está muy por debajo a la que ocurre en las ciudades del área metropolitana. Los valores pico se dan a los 1,2 y $2,2 \mathrm{~Hz}$, pero en general, la forma del espectro de ese sitio es bastante plana.

Finalmente, la estación SFRA es la que posee el nivel promedio de amplificación más bajo de las estaciones usadas en este estudio. Esto es interesante ya que en SFRA el número de eventos que se registran anualmente es muy elevado. Muchas veces se trata de eventos débiles que sólo esa estación registra aún cuando el nivel de disparo de la estación es similar al del resto de los sitios del LIS. Sería quizás recomendable prestar atención a un posible efecto topográfico que pueda estar favoreciendo a esa situación ya que el sitio se encuentra en la cima de una colina en una región muy montañosa de Costa Rica.

El factor $Q$ también se muestra en la figura 4 . Mediante un ajuste por mínimos cuadrados se determinó la ecuación del factor $Q$ en

$$
Q(f)=(231,5 \pm 0,06) f^{(0,8 \pm 0,08)}
$$

Este resultado se obtuvo invirtiendo todos los registros usados y estaciones de la tabla 1 , pero de acuerdo a la distribución de estaciones y sismos en la figura 2, se puede considerar que este resultado es representativo para la parte central y sur del país. Un nuevo cálculo de $Q$ sin usar las estaciones RMOI y PGLF, que son las más lejanas, da un nuevo resultado:

$$
Q(f)=(131,6 \pm 0,1) f^{(1,1 \pm 0,2)}
$$

en el que se puede ver que el valor es menor (lo que significa que existe mayor atenuación) que cuando se incluyen aquellas dos estaciones; sin embargo, los valores de dispersión son mayores que los de la ecuación 8. La mayor atenuación de la región central podría tener su explicación, al menos en parte, debido a que esta es una zona de fragilidad cortical primero por el vulcanismo existente (Sallares et al., 2001) y luego por el importante número de fallas que la atraviesan (Fernández \& Montero, 2002). Estas fallas han generado terremotos importantes y bastante someros como el de Piedras Negras en 1990 y el que destruyó la ciudad de Cartago en 1910 (Montero \& Miyamura, 1981).

En la parte norte, no existen suficientes registros acelerográficos para ser invertidos, pero en un estudio llevado a cabo por Christeson et al. (2000) se estimó que el valor $Q p$ debía ser tan bajo como 50 en los primeros $5 \mathrm{~km}$ de profundidad frente a las costas del pacífico norte para ajustar los modelos sintéticos a los observados. A mayores profundidades $(5$ a $15 \mathrm{~km}$ ), ellos utilizaron un valor de $Q p=500$. Christeson et al. (2000) asumieron que $Q s=0,5 Q p$, por lo que el valor es similar al que hemos calculado usando todas las estaciones, excepto que el valor obtenido en este estudio es dependiente de la frecuencia.

\section{Conclusiones}

Los efectos de sitio para la parte central de Costa Rica han sido determinadas mediante la técnica de los cocientes espectrales. Todos los sitios presentan diferentes niveles de amplificación, siendo la estación de AALJ la que posee el nivel más alto.

El valor $Q$ obtenido por la ecuación 8 podría ser representativo de la parte central-sur de Costa Rica mientras que el obtenido en la ecuación 9 de la parte central solamente. Las diferencias podrían deberse a la presencia de vulcanismo y fallamiento local del centro del país. El valor de la atenuación puede ser utilizado como indicativo de estructuras especiales que se encuentran bajo el suelo. Estructuras como una corteza altamente fracturada (presencia de muchas fallas) o zonas volcánicas activas (como lo es la parte central de Costa Rica) tenderán a dar un valor de atenuación mucho mayor, con lo cual el paso de las ondas sísmicas a través de esos cuerpos se verá fuertemente afectado. La zona del Caribe donde se encuentra RMOI y la zona sur donde se encuentra PGLF no son regiones volcánicas.

La determinación del valor de la atenuación obtenida en este estudio puede ser incorporada en trabajos de amenaza sísmica o la propagación de ondas sísmicas usando modelos numéricos y estudios de relaciones de atenuación del tipo llevado a cabo por Climent et al. (1994) y Schmidt et al. (1997). A partir de esta investigación se podrá contar con un valor de atenuación calculado con registros propios de Costa Rica y que permitirá una mejor identificación de la forma en que ésta actúa sobre los valores de aceleración pico tan importantes a la hora de diseñar estructuras sismorresistentes. 


\section{AGRADECIMIENTOS}

Este trabajo fue realizado con apoyo de la Vicerrectoría de Investigación de la Universidad de Costa Rica mediante el proyecto No. 731-A7-189. Todos los cálculos y gráficos se realizaron en el ambiente Linux utilizando el Generic Mapping Tools (Wessel \& Smith, 1998) y el programa SAC2000 (Goldstein et al., 1999). El agradecimiento se hace extensivo a la Dra. Sara Figueras Vila y un revisor anónimo por sus valiosos comentarios al presente trabajo.

\section{Referencias}

Aki, K. (1967). Scaling Law of seismic spectrum. Journal of Geophysical Research, 72: 729-740. doi:10.1029/JZ072i004p01217

Brune, J.N. (1970). Tectonic stress and the spectra of seismic shear waves from earthquakes. Journal of Geophysical Research, 75: 4997-5009.

doi:10.1029/JB075i026p04997

Christeson, G.L.; McIntosh, K.D. \& Shipley, T.H. (2000). Seismic attenuation in the Costa Rica margin wedge: amplitude modeling of ocean bottom hydrophone data. Earth and Planetary Sciences Letters, 179: 391-405. doi:10.1016/S0012-821X(00)00118-7

Climent, A.; Taylor, W.; Ciudad Real, M.; Strauch, W.; Santana, G.; Villagran, M.; Dahle, A. \& Bungum, H. (1994). Spectral strong motion attenuation in Central America. NORSAR Technical Report, August 1994, 46 pp.

DeShon, H.R, Schwartz, S.Y.; Bilek, S.L.; Dorman, L.M.; González, V.; Protti, J.M.; Flueh, E.R. \& Dixon, T.H. (2003). Seismogenic zone structure of the southern Middle American Trench, Costa Rica. Journal of Geophysical Research, 108: 2491. doi: 10.1029/2002JB002294. doi:10.1029/2002JB002294

Fernández, M. \& Montero, W. (2002). Fallamiento y sismicidad del área entre Cartago y San José, Valle Central de Costa Rica, Revista Geológica de América Central, 26: 25-37.

Goldstein, P.; Dodge, D. \& Firpo, M. (1999). SAC2000: Signal processing analysis tools for seismologists and engineers. en: IASPEI International Handbook of Earthquake and Engineering Seismology part B (Lee W.H.K.; Kanamori H.; Jennings P.C. \& Kisslinger C., eds.). Elsevier, New York, 1613-1614.

Husen, S.; Quintero, R.; Kissling, E. \& Hacker, B. (2003). Subduction-zone structure and magmatic processes beneath Costa Rica constrained by local earthquake tomography and petrological modelling. Geophysical Journal International, 155: 11-32. doi:10.1046/j.1365-246X.2003.01984.x

Iwata, T. \& Irikura, K. (1986). Separation of source, propagation, and site effects from observed S-waves. Journal of the Seismological Society of Japan, 39: 155-184.

Laporte, M. (1994). Respuesta dinámica de un depósito típico de la Ciudad de Cartago: comparación de un análisis teórico con observaciones de campo. VI Seminario Nacional de Geotecnia.

Montero, W. \& Miyamura, S. (1981). Distribución de intensidades y estimación de los parámetros focales de los terremotos de Cartago de 1910, Costa Rica, América Central. Revista del Instituto Geográfico Nacional, 2: 9-34.

Moya, A.; Schmidt, V.; Segura, C.; Boschini, I. \& Atakan, K. (1999). Empirical evaluation of site effects in the metropolitan area of San José, Costa Rica. 1999. Abstracts Volume, Ninth International Conference on Soil Dynamics and Earthquake Engineering. Bergen, Norway.

Moya, A. \& Irikura, K. (2003). Estimation of site effects and $Q$ factor using a reference event. Bulletin of the Seismological Society of America, 93: 1730-1745. doi:10.1785/0120020220

Pacheco, J.F.; Quintero, R.; Vega, F.; Segura J.; Jiménez, W. \& González, V. (2006). The Mw 6.4 Damas, Costa Rica, Earthquake of 20 November 2004: Aftershocks and Slip Distribution. Bulletin of the Seismological Society of America, 96: 1332-1343.

doi:10.1785/0120050261

Protti J.M.; MCnally, K.; Pacheco, J.; González, V.; Montero, C.; Segura, J.; Brenes, J.; Barboza, V.; Malavassi, E.; Güendel, F.; Simila, G.; Rojas, D.; Velasco, A.; Mata, A. \& Schillinger, W. (1995): The March 25, $1990(\mathrm{Mw}=7,0, \mathrm{ML}=6,8)$ earthquake of the entrance of Nicoya Gula, Costa Rica: Its prior activity, foreshocks, aftershocks, and triggered seismicity. Journal of Geophysical Research, 100: 20.345-20.358

Ramírez, R.; Santana, G. \& Chacón, O. (1996). Mapa de amplificación sísmica del Valle Central, Costa Rica. Revista Geológica de América Central, 19: 37-55.

Sallares, V.; Dañobeitia J.J. \& Flueh, E.R. (2001). Lithospheric structure of the Costa Rican Isthmus: Effects of subduction zone magmatism on an oceanic plateau. Journal of Geophysical Research, 106: 621-643. doi:10.1029/2000JB900245

Schmidt, V.; Moya, A.; Climent, C.; Rojas, W.; Boschini, I.; Lindholm, C.; Nadim, F.; Bungun, H. \& Atakan, K. (2005). Microzonificación Sísmica de San José, Costa Rica. CD-ROM, Editorial Universidad de Costa Rica, San José, Costa Rica.

Schmidt, V.; Dahle, A. \& Bungum, H. (1997). Costa Rican Spectral Strong Motion Attenuation. NORSAR Technical Report (RONDICA Project), Kjeller, Noruega. 47 pp.

Steidl, J.H.; Tumarkin, A.G. \& Archuleta, R.J. (1996). What is a reference site? Bulletin of the Seismological Society of America, 86: 1733-1748.

Wessel, P. \& Smith, W.H.F. (1998). New, improved version of the Generic Mapping Tools released, Eos, Transactions, American Geophysical Union, 79: 579. doi:10.1029/98EO00426

Recibido el 23 de mayo de 2008 Aceptado el 16 de enero de 2009 Publicado online el 28 de abril de 2009 